\title{
Impact of dietary nutrient intake and physical activity on body composition and growth in Indian children
}

\author{
Anuradha V. Khadilkar ${ }^{1}$, Shashi A. Chiplonkar ${ }^{1}$, Neha A. Kajale${ }^{1}$, Veena H. Ekbote ${ }^{1}$, Lavanya Parathasarathi ${ }^{1}$, Raja Padidela $^{2}$ \\ and Vaman V. Khadilkar ${ }^{1}$
}

BACKGROUND: To investigate interrelationships of dietary composition and physical activity (PA) with growth and body composition (BC) in urban Indian school children.

METHODS: A cross-sectional study was performed in 4,747 Indian school children (2,623 boys) aged 3-18 years. Weight, height, BC by body impedance analyzer, PA and dietary food intakes by questionnaire method were recorded.

RESULTS: Mean daily energy and protein intakes as recommended dietary allowance were significantly lower in both boys and girls $(P<0.01)$ above 6 years, with 55\% reduction in micronutrient intakes in older children. When compared with World Health Organization references, lower heights in pubertal boys and girls were related to dietary energy and protein intake. Multiple regression analysis showed positive association of height for age $z$-scores (HAZ) with midparental height $z$-scores $(\beta=0.45, P=0.0001)$ and protein density $(\beta=0.103, P=0.014)$. HAZ was negatively associated with inactivity $(\beta=-0.0001, P=0.049)$ in boys and girls $\left(R^{2}=0.104, P<0.01\right)$. Further, body fat percentage was negatively correlated with moderate or light activity and antioxidant intakes $(P<0.01)$ but not with dietary fat intake. Percentage muscle mass was positively correlated with moderate activity and negatively with inactivity $(P<0.05)$.

CONCLUSION: Adequacy of protein and antioxidant intakes, reducing inactivity and increasing moderate activity are essential for optimal growth and body composition in Indian children.

A $s$ a result of the nutrition transition, developed as well as developing countries such as India are facing the dual challenge of childhood obesity and nutrient deficiencies. Changing dietary practices such as consumption of fast foods, sweetened beverages, and energy-dense food increases the risk of nutrient imbalance, thus leading to childhood obesity. Further, the global prevalence of overweight and obesity in youth is on the rise (1) including in India (2).

It has also been observed that Asian Indian children and adolescents have a higher body fat percentage at lower body mass index (BMI) values (3). Available evidence also suggests that Indian children and adolescents have inadequate dietary intakes of energy and protein but not fat $(4,5)$. High-fat intake of Indian youth may be one of the reasons for their adiposity, since fats have greater energy density of $9 \mathrm{kcal} / \mathrm{g}$ than that of proteins and carbohydrates $(4 \mathrm{kcal} / \mathrm{g})$ and the fat content of the diet has been reported to have an effect on body fat as a function of the effect of dietary fat on energy intake (6). However, evidence also suggests an inverse relationship of dietary carbohydrate intake $(6,7)$ and both a positive and inverse relationship of protein intake with body fat $(8,9)$. Further, these imbalances in nutrient intake may, together with resulting in adiposity/obesity, also affect growth in adolescence.

Nutrition and growth are integrally related; inadequate nutrient intake during growing years may reduce or arrest linear growth and also act as a stepping stone for diseases in later life (10). It has been recently observed that growth in Indian children remains less than optimum in comparison with children from western countries (11). This suggests that "more than adequate fat intake" alone does not guarantee adequacy of energy intake. This is supported by studies reporting dietary deficiencies of energy, protein, vitamins, and minerals in Indian children and adolescents $(5,12)$. Thus, a balance in diet composition appears to be more important than the intake of individual nutrients to achieve optimal growth and reduce the risk of obesity.

A positive energy balance also implies that dietary energy intake is higher than the energy expenditure. Lack of physical exercise and sedentary lifestyle are the leading factors for obesity in both adults and children $(13,14)$. Therefore, a highfat diet with low physical activity would necessarily result in a positive energy balance, thus promoting adiposity even after considering the energy cost of weight gain (in growing years this is $1 \%$ of the total energy requirement) (15). However, associations of dietary fat intake and energy intake with percentage of body fat are not consistently observed in studies $(7,16)$. Moreover, the effects of growth and development may interact with diet composition or physical activity, or both, in ways that are not yet well understood.

Growth and Endocrine Unit, Hirabai Cowasji Jehangir Medical Research Institute, Jehangir Hospital, Pune, India; ${ }^{2}$ Department of Paediatric Endocrinology, Royal Manchester Children's Hospital, Manchester, UK. Correspondence: Anuradha V. Khadilkar (anuradhavkhadilkar@gmail.com)

Received 4 January 2017; accepted 9 October 2017; advance online publication 17 January 2018. doi:10.1038/pr.2017.322 


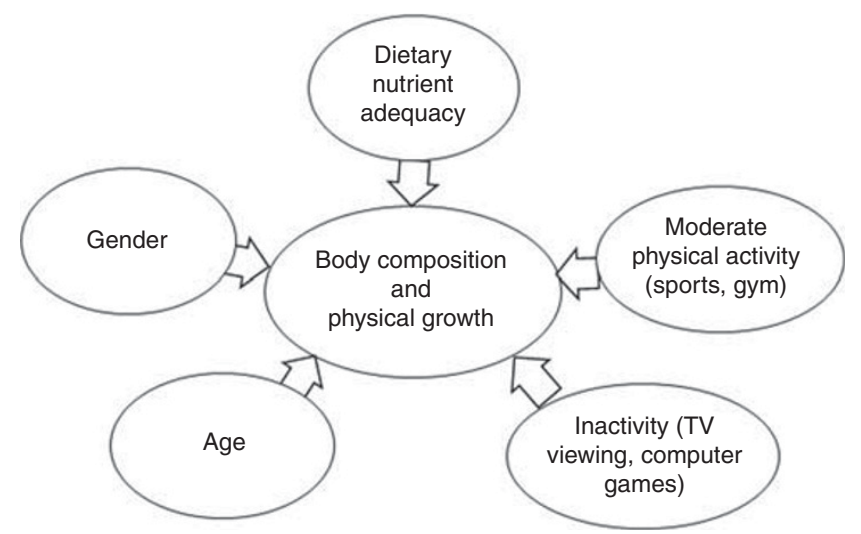

Figure 1. Interrelationships of growth and body composition with age, gender, and lifestyle factors.

Several studies have shown that children who spend more time watching television have a higher BMI and body fat percentage and are less physically active $(17,18)$. On the other hand, urban Asian Indian adolescents who participate regularly in outdoor games have been shown to have a lower prevalence of obesity with the risk being three times higher in those not participating in outdoor games (19).

Taken together, urban Asian Indian children and adolescents have changing dietary practices and an increasingly sedentary lifestyle which puts them at a higher risk of obesity and disturbances in growth. Studying interrelationships between nutrient adequacy, physical activity, body composition, and growth may help in understanding the means by which growth and body composition may be optimized. These interrelationships may be masked with exogenous factors such as socioeconomic status (SES). Girls from low SES have been shown to have significantly higher nutrient deficiencies than girls from higher SES group (5). Thus, studying these relationships where exogenous factors can be minimized is important. Therefore, our aim was to study associations of nutrient intake and physical activity with body composition and growth in Asian Indian children (Figure 1) from higher SES and to compare their growth with international growth data. Our specific objective was to investigate the linkages between nutrient intakes and physical activity with body composition and growth in 3-18-year-old apparently healthy Indian children.

\section{METHODS}

\section{Selection of Participants}

This was a part of a multicenter cross-sectional study in 3-18-yearold Indian children (July 2010 to January 2012). To capture all geographical locations of India, five zones as suggested by the Indian Academy of Pediatrics were considered for data collection (20). One major city, based on per capita income from each of the five zones (Chennai-South India, Delhi-North India, Kolkata-East India, Pune-West India, and Raipur-Central India) was selected. A list of schools catering to children of socioeconomically well-off families was made for each city with the help of local coinvestigators. Six schools were then randomly selected from each city and were approached for permissions to carry out measurements.
On a random sample of 4,747 school children (2,623 boys), data on anthropometry, diet (1-day recall) (21), body composition by Tanita bioimpedance analyzer (Tanita Corporation, Tokyo, Japan) (22), and physical activity by a standardized questionnaire (23) were recorded. The study was approved by the Ethics Committee of the Hirabai Cowasji Jehangir Medical Research Institute. Written institutional consent was obtained from the principals of all schools and written informed consent was obtained from parents before the study was commenced.

Clinical examination (including a general physical examination and an assessment of the cardiovascular and respiratory system) was carried out by pediatricians to assess health status of the children. Children with major illness, or any known condition affecting growth were excluded from the study.

\section{Anthropometric Measurements}

Standing height was measured with a portable stadiometer (Leicester Height Meter; Child Growth Foundation, London, UK; range, 60$207 \mathrm{~cm}$ ) with the child barefoot. Weight was measured with a portable electronic scale (Salter India, Faridabad, India) accurate to $100 \mathrm{~g}$ (calibration performed using standard weights). BMI was calculated, and height for age $z$-scores (HAZ), weight for age $z$-scores (WAZ), and BMI for age $z$-scores (BAZ) were computed using Indian reference data as well as the World Health Organization (WHO) references $(20,24)$. To account for changes in anthropometric measures associated with increase in age and associated pubertal changes, as well as the later growth spurt in boys, the following age groups were defined: boys A (3-5 years), B (6-12 years), C (13-15 years), and D (16-18 years), girls as A (3-5 years), B (6-9 years), C (10-14 years), and D (15-18 years) (25).

\section{Body Composition}

Body composition was assessed using Bioelectrical Impedance Analyzer (BIA), Tanita Model BC-420MA after a minimum of $3 \mathrm{~h}$ of fasting and voiding before measurements (10 AM onward) (22). Body composition of the subjects was assessed on the BIA by measuring the impedance to electric current passed through the body in a standing position. The body composition parameters assessed were fat percentage, fat mass, fat-free mass, total body water, bonefree lean tissue mass, and bone mass. Body composition measurements were tested for test-retest reliability on a pilot sample of 30 subjects separately by measuring them on BIA at two different time points within a day. Reliability coefficient was significant for the body fat percent, fat mass, fat-free mass, and muscle mass (intraclass correlation coefficient $=0.96, P<0.001$ ). Using the body fat reference curves by McCarthy et al. (26), individual child's percentile of body fat $\%$ was computed by interpolation and extrapolation techniques.

\section{Dietary Intake Assessment}

Information on children's daily food intakes was collected by a selfadministered 24-h diet recall on one weekday (21) in English language. Choices of common food items consumed for each region were provided in the questionnaire to enable accurate reporting of diet recall. After the dietary data were collected, detailed recipes of most commonly consumed food items were recorded from parents or caregivers for each region. The portion size was obtained by the average of actual weights of one serving of each food item from their households. This was done for each of the recorded food items. Moisture and the nutrient contents of the newly developed recipes were estimated. Based on moisture correction, nutrient contents for the modifications were estimated. Further, in a subset of households, to estimate the portion sizes of foods, the average cups and measures of each region were converted to standard cups and measures. The portion size was converted to weights by weighing each food item in the household.

Validation of the dietary intakes by 1-day dietary recall (reported by parents) against the average of dietary intakes from 24-h, 3-day diet recalls (two nonconsecutive days and a holiday) by interview method was conducted in a subset of 20 children at each center. 
One-day diet recall questionnaire was self-administered by the parents of the children, whereas the 3-day diet recalls were collected by a trained nutritionist by using "multiple pass approach" $(27,28)$. The reliability coefficient was significant for all the food groups (intraclass correlation coefficient ranged from 0.81 to 0.98 for different food intakes, $P<0.05$ ).

Daily nutrient intakes were estimated with the use of C-Diet software version 2.0 (29), which comprises of nutritive values of cooked foods (30-32) and raw food database of National Institute of Nutrition, India (33) and USDA (32). Moisture and the nutrient contents of the new recipes were estimated. Based on the moisture correction, nutrient contents for the new recipes from all the regions were estimated and used for computing nutrient intakes.

For assessing the nutrient adequacy of the diet, percentage intake of recommended dietary allowance (RDA) for Indians as given by the Indian Council of Medical Research was calculated for all the nutrients (33). To examine the relationship of dietary nutrient intakes and growth in terms of HAZ, children were categorized in 1year age groups.

\section{Physical Activity}

Physical activity was assessed using the QAPACE school children questionnaire (23) by interview and school schedules. The questionnaire was adapted for Indian children and adolescent's lifestyle since commonly played sports and the type of religious activities in India were different, and sections pertaining to these activities were modified from the QAPACE questionnaire. Time spent by the participant in sports activities (jogging, swimming, dancing, brisk walking, school sports/games, and playing outdoor games) was considered as moderate activity (34). For inactivity, the total amount of time spent in watching television, using laptops or mobile phones for playing games, chatting, and other sedentary behavior was estimated. The reliability analysis comparing responses by selfadministration of questionnaire and by face-to-face interview in a subset of 30 children revealed the intraclass correlation coefficient of 0.94 for inactivity and 0.99 for moderate activity $(P<0.01)$.

\section{Statistical Analysis}

Statistical analysis was performed using SPSS (version 21.0, 2012, SPSS, Chicago, IL). Normality of the outcome variables was tested using Kolmogorov-Smirnov test. For non-normal variables, nonparametric tests were used. Comparison of diet and body composition of different age-sex groups was done using one-way ANOVA with post hoc multiple-comparison tests. Pearson's product moment correlation coefficient or Spearman's rank correlation coefficient were used to estimate the association of dietary and anthropometric variables. Multiple linear-regression analysis was performed to examine the simultaneous effect of diet and physical activity on body composition parameters and growth.

\section{RESULTS}

Data on the anthropometric and body composition parameters of 4,747 (2,623 boys) of apparently healthy children (without the history of any chronic disease) aged 3-18 years are described in four age groups (Table 1). Except the first age group, boys had higher mean values of height and weight than girls $(P<0.05)$. As per the current Indian growth references, mean HAZ was $0.08 \pm 0.02$ in boys and $0.14 \pm 0.02$ in girls, indicating that the majority of children and adolescents $(85.9 \%)$ were within the reference range for height for age. Mean WAZ was $0.07 \pm 0.02$ in boys and $0.14 \pm 0.02$ in girls and mean BMIZ was $0.04 \pm 0.02$ in boys and $0.08 \pm 0.02$ in girls. As per the WHO cutoffs for weight, $80.6 \%$ children were within the reference range, $15.7 \%$ were overweight, and $3 \%$ were obese (35). Body fat percentage was higher and

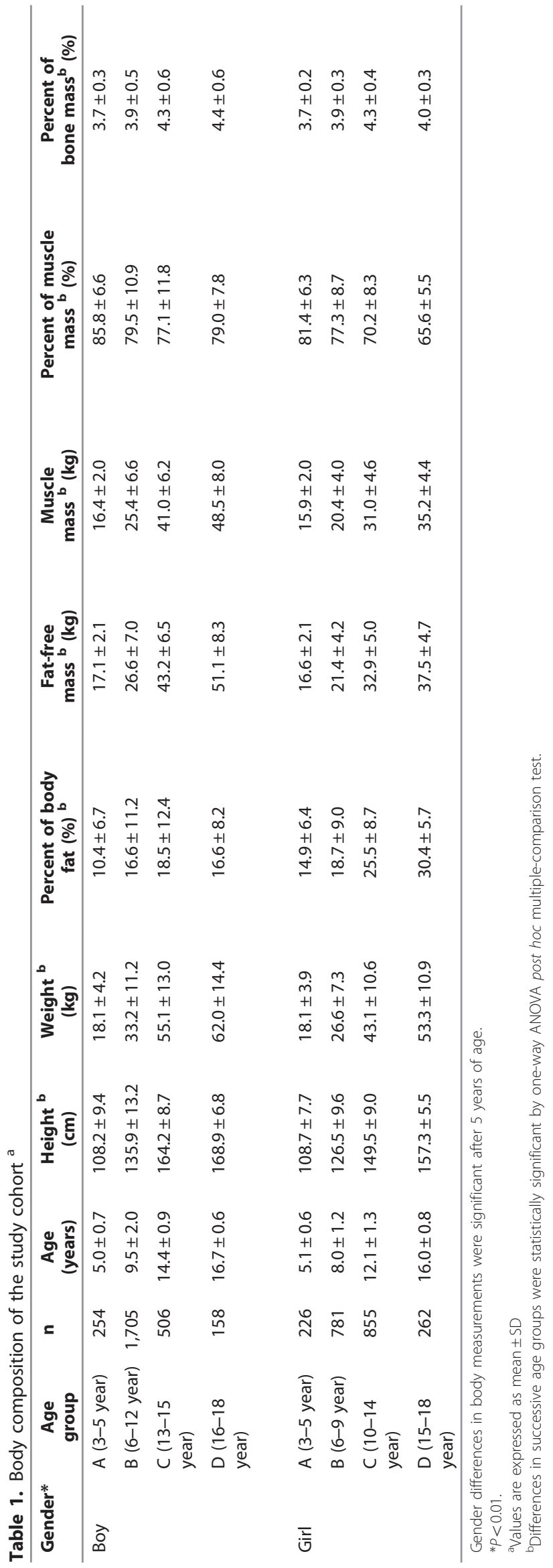




\section{Articles | Khadilkar et al.}

percentage of muscle mass was lower in girls than boys in all age groups $(P<0.01)$ (Table 1$)$.

\section{Physical Activity}

In boys, the average time spent in moderate physical activity was more in older age groups (A: $328 \pm 20 \mathrm{~min}$ per week vs. D: $417 \pm 19$ min per week), whereas in girls, moderate activity was lesser in older age groups (A: $324 \pm 18 \mathrm{~min}$ per week vs. D: $244 \pm 15$ min per week $)(P<0.01)$. Inactivity in boys was more in older age groups (A: $462 \pm 20 \mathrm{~min}$ per week vs. D: $610 \pm 26$ min per week) $(P<0.05)$. However, minutes spent in inactivity were similar in younger and older girls (A: $442 \pm 21$ min per week vs. D: $508 \pm 25$ min per week) (Figure 2).

\section{Diet Composition}

Mean nutrient intakes in boys and girls were similar in age group A (3-5 years) (Table 2). In later age groups, boys had

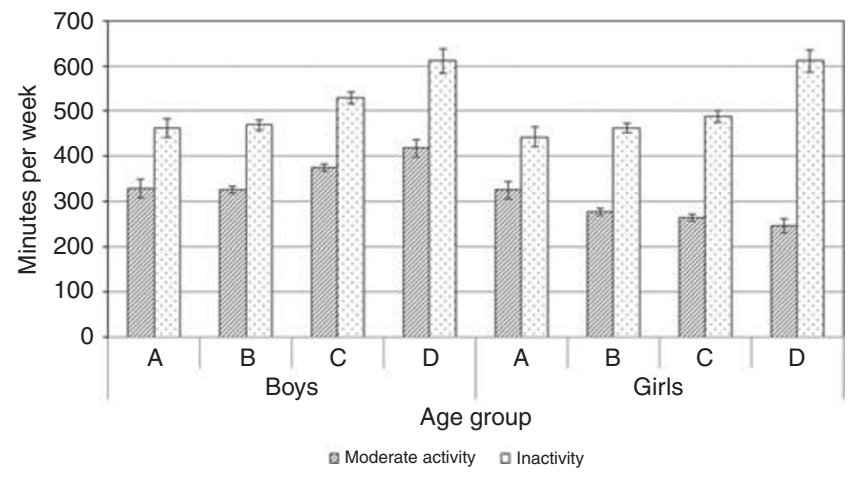

Figure 2. Weekly physical activity across gender and age groups. significantly higher intakes of the majority of nutrients $(P<0.05)$. There was a gradual rise in absolute nutrient intakes from lower age group A to higher age group D. Mean percent calories from fats $(24.2 \pm 5.1 \%)$ and carbohydrates $(65.2 \pm 5.8 \%)$ remained similar in all age-sex groups.

\section{Adequacy of Nutrient Intakes and Growth Status Across Age Groups}

Energy and protein intakes expressed as percent RDA showed significant reduction after 6 years, in both boys and girls $(P<0.01)$ (Figure 3a,b). Mean daily micronutrient intakes in boys were $\sim 83 \%$ of RDA till 9 years of age and then gradually reduced to $55 \%$ up to 18 years. In girls, mean daily micronutrient intakes were lower than boys and were around $79 \%$ up to 9 years of age and declined to $44 \%$ at 18 years of age. HAZ in the recruited children using Indian references were higher than those using WHO reference charts (24) throughout the age range. However, both the HAZ scores were closer during 7-11 years in boys and 7-10 years in girls (Figure 3a,b). In boys $\sim 14$ years, HAZ by Indian references showed an increase, after which there was a decline till 18 years of age (Figure 3a). On the contrary, HAZ by WHO references showed a sharp decrease from 11 years of age. The reduction in dietary energy and protein intake goes hand in hand with HAZ by WHO after 12 years of age, but not so with HAZ by Indian references.

In girls, the peak at 12 years in $\mathrm{HAZ}$ Indian references and the decline in HAZ by WHO after 10 years was smaller than that in boys (Figure 3b). Corresponding downward trends in energy and protein intakes were sharper than those in boys.

Table 2. Mean daily nutrient intakes in boys and girls across age groups

\begin{tabular}{|c|c|c|c|c|c|c|c|c|}
\hline \multirow[t]{4}{*}{ Nutrient intake per day ${ }^{a^{*}}$} & \multicolumn{4}{|c|}{ Boys } & \multicolumn{4}{|c|}{ Girls } \\
\hline & \multicolumn{4}{|c|}{ Age group } & \multicolumn{4}{|c|}{ Age group } \\
\hline & A & B & C & D & A & B & C & D \\
\hline & 3-5 years & 6-12 years & 13-15 years & $16-18$ years & 3-5 years & 6-9 years & 10-14 years & 15-18 years \\
\hline Energy (kcal) & $1,457 \pm 485$ & $1,668 \pm 559$ & $1,776 \pm 620$ & $1,688 \pm 623$ & $1,396 \pm 449$ & $1,522 \pm 527$ & $1,659 \pm 615$ & $1,402 \pm 554$ \\
\hline Protein (g) & $41.6 \pm 14.2$ & $46.8 \pm 16.3$ & $49.0 \pm 17.9$ & $45.6 \pm 18.1$ & $40.2 \pm 13.7$ & $43.0 \pm 16.2$ & $45.2 \pm 17.0$ & $36.5 \pm 13.8$ \\
\hline Fat (g) & $38.1 \pm 15.1$ & $45.7 \pm 18.6$ & $46.1 \pm 19.3$ & $42.6 \pm 20.2$ & $37.8 \pm 16.1$ & $41.3 \pm 7.5$ & $43.8 \pm 19.7$ & $36.8 \pm 19.5$ \\
\hline Zinc (mg) & $4.8 \pm 1.8$ & $5.5 \pm 2.0$ & $6.1 \pm 2.6$ & $5.9 \pm 2.3$ & $4.5 \pm 1.6$ & $4.9 \pm 2.1$ & $5.2 \pm 2.1$ & $4.4 \pm 1.8$ \\
\hline Iron (mg) & $7.3 \pm 3.1$ & $8.7 \pm 3.7$ & $9.4 \pm 4.1$ & $9.2 \pm 4.0$ & $6.9 \pm 3.1$ & $8.0 \pm 3.6$ & $8.2 \pm 3.7$ & $7.0 \pm 3.5$ \\
\hline Copper (mg) & $1.1 \pm 0.4$ & $1.4 \pm 0.5$ & $1.5 \pm 0.6$ & $1.4 \pm 0.6$ & $1.1 \pm 0.4$ & $1.2 \pm 0.5$ & $1.4 \pm 0.6$ & $1.2 \pm 0.5$ \\
\hline Vitamin C (mg) & $40 \pm 22$ & $45 \pm 27$ & $43 \pm 29$ & $37 \pm 25$ & $39 \pm 22$ & $45 \pm 29$ & $47 \pm 32$ & $35 \pm 23$ \\
\hline$\beta$-carotene $(\mu \mathrm{g})$ & $1,316 \pm 829$ & $1,662 \pm 1,070$ & $1,821 \pm 1,245$ & $1,876 \pm 1,290$ & $1,285 \pm 786$ & $1,612 \pm 1,140$ & $1,717 \pm 1,112$ & $1,461 \pm 1,062$ \\
\hline Folic acid $((\mu \mathrm{g})$ & $76.9 \pm 39.8$ & $85.2 \pm 38.4$ & $92.9 \pm 42.1$ & $91.8 \pm 45.2$ & $70.8 \pm 30.7$ & $80.0 \pm 39.3$ & $82.8 \pm 40.8$ & $73.4 \pm 39.2$ \\
\hline
\end{tabular}



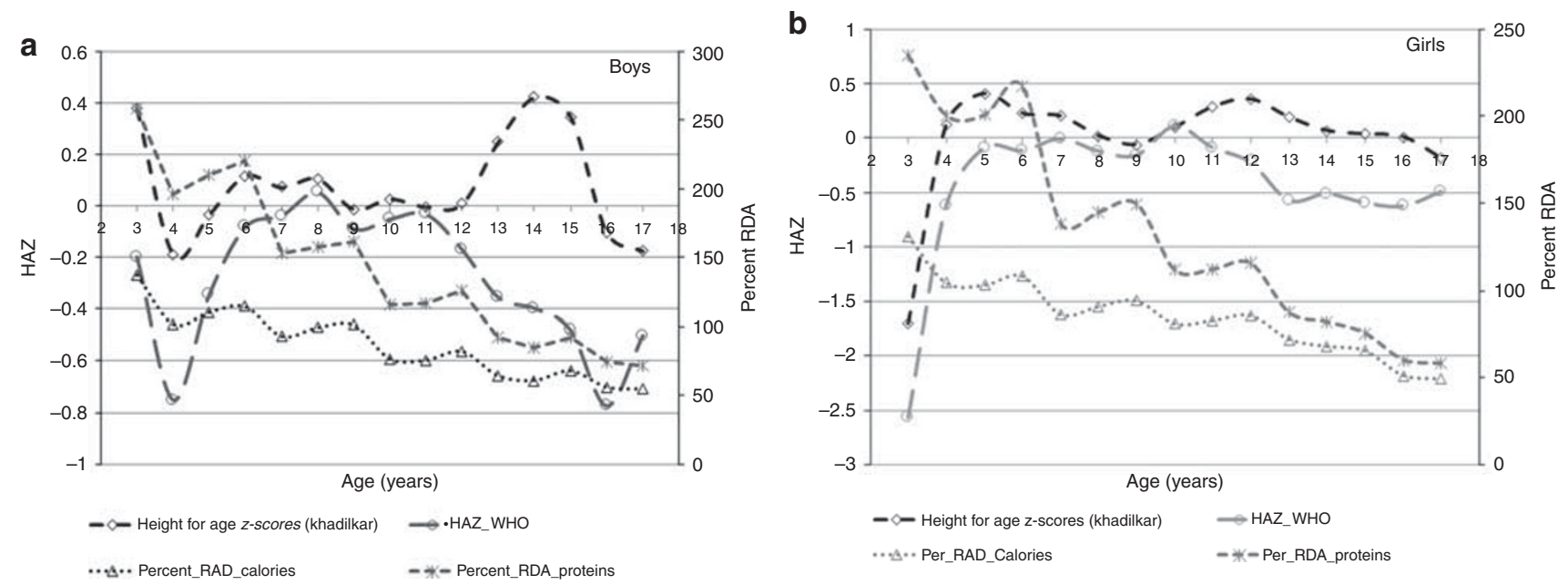

Figure 3. ( $\mathbf{a}$ and $\mathbf{b}$ ) Dietary energy and protein adequacy and height $z$-scores across age groups in boys and dietary energy and protein adequacy and height $z$-scores across age groups in girls.
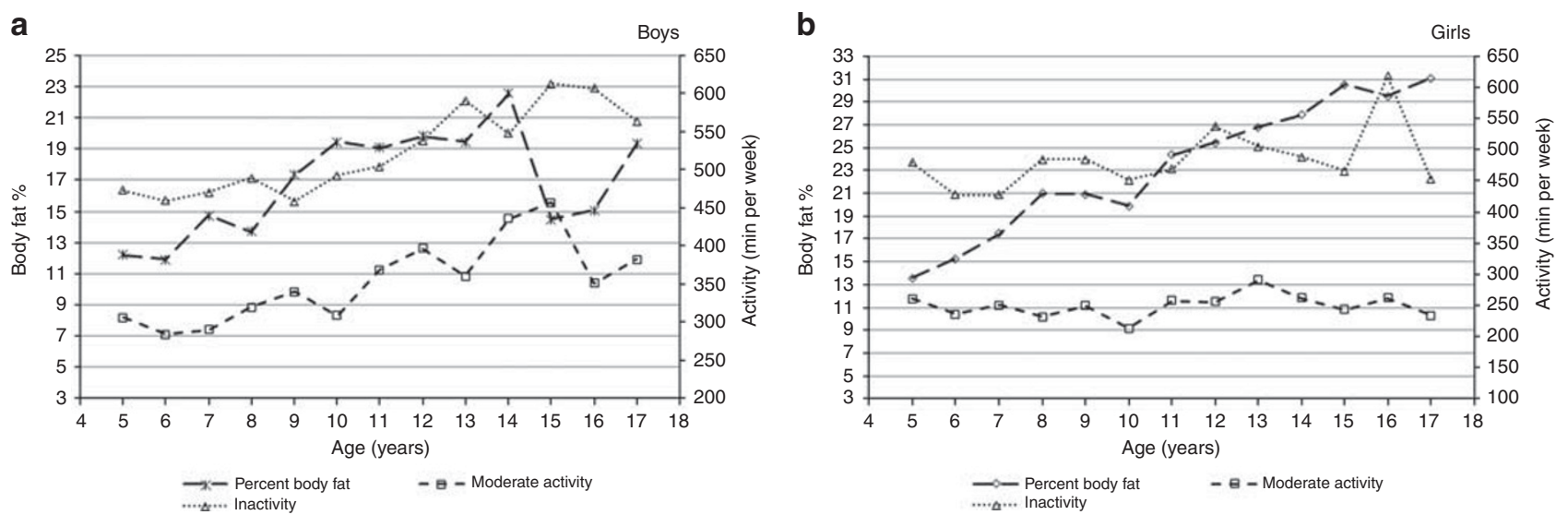

Figure 4. (a and $\mathbf{b}$ ) Percent of body fat and physical activity in boys and percent of body fat and physical activity in girls.

\section{Linkages of Diet, Physical Activity, and Body Composition}

Correlations between percentage of body fat and daily energy intake, fat intake, and percentage of dietary energy from fat and carbohydrates in all children were low $(r=-0.02$ to $-0.04)$ and remained low and statistically nonsignificant $(P>0.1)$ even after adjusting for age in both boys and girls $(P>0.1)$. Moreover, correlations of body fat percentile estimated by McCarthy reference centiles (26), and dietary energy or fat intakes were also found to be low and statistically not significant in all children. However, in girls, daily antioxidant intake showed a negative correlation ( $r=-0.14, P<0.01)$ with body fat percentile.

Muscle mass percentage and bone mass showed significant correlation with energy $(r=0.14)$ and protein intakes $(r=0.16) \quad(P<0.05)$, which did not remain after adjusting for age, underlining the influence of age on diet.

In yearly age groups, moderate activity in minutes per week was high in higher age groups $(P<0.01)$. Inactivity in minutes per week was higher than the moderate activity at all ages from 3 to 17 years. In girls, moderate activity was lower than that in boys and much less than their inactivity throughout the age range (Figure 4a,b). Percent of body fat and inactivity was closer till 14 years of age in boys. Around 15 years of age, there was a decline in percent of body fat with a corresponding rise in moderate activity. In girls, percent of body fat and inactivity was closer, although the moderate activity remained the same throughout till 18 years.

A multiple linear-regression analysis for body fat percentile revealed that in boys, there was a positive association of inactivity $(\beta=0.012, P=0.031)$, and negative association of moderate activity $(\beta=-0.078, P=0.047)$ with body fat percentile $\left(R^{2}=0.22, P<0.01\right)$. However, in girls, a negative association of antioxidant micronutrient intakes (\% of energy) $(\beta=-3.46, P=0.024)$ and light activity $(\beta=-0.07, P=0.15)$ with body fat percentile $\left(R^{2}=0.19, P<0.05\right)$ was observed.

Multiple linear regression for percentage of muscle mass indicated a positive association of moderate $(\beta=0.031$, $P=0.095)$ and light activity $(\beta=0.01, P=0.025)$ and negative 


\section{Articles | Khadilkar et al.}

association with inactivity $(\beta=-0.002, P=0.047)$ in boys. In girls, a positive association of moderate $(\beta=0.006, P=0.024)$ and light activity $(\beta=0.02, P=0.0001)$ with percent of muscle mass was observed. These linkages remained significant after adjusting for age. However, no associations with dietary intakes were found to be significant.

Multiple linear-regression analysis for HAZ as a growth indicator suggested a positive association of mid-parental height $(\mathrm{MPH}) z$-score $(\beta=0.45, P=0.0001)$, protein density $(\beta=0.103, \quad P=0.014)$, and a weak negative association of inactivity $(\beta=-0.0001, P=0.049)$ in boys and girls $\left(R^{2}=0.104, P<0.01\right)$.

\section{DISCUSSION}

The study population was apparently healthy children and adolescents from the urban upper socioeconomic class covering five zones of India. Dietary energy and protein intakes were found to be adequate in the early years in both boys and girls; however, there was a decline in energy adequacy and dietary protein intake around 10-11 years which is the usual age of the adolescent growth spurt. This may hamper achieving target height in these youths. Micronutrient intake also reduced around this age; this may also potentially impact growth, development, and health.

In line with intakes in early years, the HAZ scores as per the Indian references remained around zero till 12 years in boys and 10 years in girls. However, from 12 to 16 years of age, HAZ scores were higher in both boys and girls and were below zero at 16 years in both genders. While corresponding with Indian HAZ scores, HAZ scores based on WHO data showed a gradual decline from peripubertal age in boys and girls, suggesting that Indian children and adolescents fell short of WHO reference population along with reduction in total energy and protein intake. Pubertal stage may confound the relationship of age with growth and body composition. Thus, lower HAZ may also be explained on the basis of later puberty; however, studies suggest that timing of puberty in urban-affluent Indian children is similar to that in children in developed countries (36). Our data thus suggest that dietary inadequacy during adolescent years may be a factor contributing to suboptimal growth in Indian children and adolescents compared with WHO data. With nutrient adequacy, peak HAZ would possibly have been higher than the current value.

The finding that MPH was a significant predictor of HAZ underlines the contribution of genetic factors toward final height either in isolation or by modulating nutrient intake and onset of puberty. In line with our results, $\mathrm{MPH}$ has been shown to be correlated with a child's current height and MPH has been used to correct for genetic potential and to identify nonfamilial short stature (37).

Body fat increases with age until puberty. At puberty, sex hormones induce a pronounced sexual dimorphism: boys gain proportionately more muscle and lean tissue as compared with fat, and girls lay down fat as a natural part of the ontogeny of their sexual and reproductive physiology
(26). Our data also exhibit an increase in body fat percentage till 13 years of age and then a decline after 14 years in boys but not so in girls.

Although dietary energy and protein intakes were lower than the RDA, they were not significantly associated with body fat or muscle mass percentage. Our results did not exhibit any significant relationship between fat intake as a proportion of energy intake and percentage of body fat. This was further corroborated by the lack of any significant difference between mean percentage of body fat across the dietary energy and fat intake quartiles for boys and girls separately $(P>0.1)$. Similar findings have been previously reported in preschool children and adolescents (38). Dietary carbohydrate intake as a percentage of energy intake is often inversely related to body fat percentage in overweight or obese children $(6,7)$. This is possibly due to lower energy density of carbohydrate than fat. However, in the present study, there was no association between carbohydrate intake and percentage of body fat. The possible reason could be energy intakes of children above 6 years of age in the current study were less than RDA, indicating that intake of carbohydrate was also not in excess amounts to be stored as body fat. This is in agreement with other studies on school children (38). Upon further examination, no significant associations were obtained in the subsets of overweight or obese children in the current study cohort.

In an earlier cross-sectional study, we have found that girls with short stature (HAZ $<-2)$ had significantly lower micronutrient intakes in comparison with the required RDA (5). In the present study, micronutrient intakes were found to be low in both boys and girls throughout childhood years and were $44-68 \%$ of the RDA around puberty.

Many cross-sectional (39) and longitudinal (40) studies indicate that increased physical activity and decreased sedentary behavior are protective against relative weight and fatness gains over childhood and adolescence. Moreover, high adiposity and low moderate activity have been found to pose cardiometabolic risk in youth (39). In the present study, $63 \%$ of $6-12$-year-old boys ( $45 \%$ girls) and $73 \%$ older boys ( $46 \%$ girls) were engaged in moderate activity for $45 \mathrm{~min}$ per day for more than 3 days per week. Moderate activity showed an inverse relation with body fat percentage in both boys and girls. Around $47 \%$ boys and $41 \%$ girls spent more than 420 min per week on TV viewing/computer time. Our results also demonstrate that inactivity has positive association and moderate activity has negative association with body fat.

The strength of our study is that we have measured interrelationships of body composition and growth with diet and physical activity over a wide age range of Indian children from five zones of India. Data were collected by the same team at all places, and detailed record of physical activity and diet was performed. These data may help to optimize growth and body composition in Indian children. However, being a cross-sectional study, our study can only provide information on the possible associations among diet, physical activity with growth, and body composition and cannot describe causal 


\section{Effect of diet on $B C$ in Indian children $\quad$ Articles}

relationships of adiposity with diet and activity. The other limitation of our study is that diet and activity assessments were performed using 1-day recall and questionnaire method which can offer short-term measures and may not be representing the participant's habitual diet or activity level. The 1-day recalls were, however, validated against 3-day recalls. Further, growth and pubertal status play a significant role in body composition measurements; however, since this was a school-based study, we were not able to assess sexual maturity and genetic factors.

In summary, our data demonstrate that total dietary energy and fat intakes as also percent energy from fat or carbohydrates were not related to the body fat or muscle mass percentage. Reduced nutrient intakes with a high-fat intake contribute to the higher body fat percentage coupled with suboptimal growth in Indian children. Adequacy of protein and antioxidant intakes, reducing inactivity, and increasing moderate activity are essential factors for optimal growth and body composition in Indian children.

Disclosure: No financial assistance was received in support of the study. The authors declare no conflict of interest.

\section{REFERENCES}

1. World Health Organization Global Strategy on Diet, Physical Activity and Health: Childhood Overweight and Obesity, 2012. Available at http:// www.who.int/dietphysicalactivity/childhood/en/.

2. Pandit D. Overweight and obesity prevalence and body mass index trends in Indian children. Int J Pediatr Obes 2010;6:1-9.

3. Pandit D, Khadilkar A, Khadilkar V. Clinical medicine: pediatrics body fat percentages by dual-energy X-ray absorptiometry corresponding to body mass index cutoffs for overweight and obesity in Indian children. Cin Med Pediatr 2009;3:1-7.

4. Tupe R, Chiplonkar SA. Diet patterns of lactovegetarian adolescent girls: need for devising recipes with high zinc bioavailability. Nutrition [Internet] 2010;26:390-8 Available at http://dx.doi.org/10.1016/j.nut.2009.05.018.

5. Chiplonkar S, Khadilkar A, Pandit-Agrawal D, Kawade R. Influence of micronutrient status and socioeconomic gradient on growth indices of 2-18-year-old Indian girls. J Pediatr Endocrinol Metab 2013;26:825-32.

6. Gazzaniga JM, Burns TL. Relationship between diet composition and body fatness, with adjustment for resting energy expenditure and physical activity, in preadolescent children. Am J Clin Nutr [Internet] 1993;58: 21-8 Available at http://www.ncbi.nlm.nih.gov/pubmed/8317384 (cited 2 January 2017).

7. Tucker LA, Seljaas GT, Hager RL. Body fat percentage of children varies according to their diet composition. J Am Diet Assoc [Internet] 1997;97: 981-6 Available from http://linkinghub.elsevier.com/retrieve/pii/ S000282239700237X (cited 2 January 2017).

8. Vinknes KJ, de Vogel S, Elshorbagy AK, et al. Dietary intake of protein is positively associated with percent body fat in middle-aged and older adults. J Nutr [Internet] 2011;141:440-6 Available at http://www.ncbi.nlm.nih.gov/ pubmed/21248195 (cited 2 January 2017).

9. Ledoux TA, Watson K, Barnett A, Nguyen NT, Baranowski JC, Baranowski T. Components of the diet associated with child adiposity: a cross-sectional study. J Am Coll Nutr [Internet] 2011;30:536-46 Available at http://www.ncbi.nlm. nih.gov/pubmed/22331689 (cited 2 January 2017).

10. Srilaxmi B. Dietetetics. Delhi, India: New Age International (P) limited, 2005.

11. Khadilkar V, Khadilkar A. Revised Indian Academy of Pediatrics 2015 growth charts for height, weight and body mass index for 5-18-year-old Indian children. Indian J Endocrinol Metab [Internet] 2015;19:470 Available at http://www.ijem.in/text.asp?2015/19/4/470/159028.

12. Srihari G, Eilander A, Muthayya S. Nutritional status of affluent Indian school children: what and how much do we know? Indian Pediatr
[Internet] 2007;44:204-13 Available at http://www.ncbi.nlm.nih.gov/ pubmed/17413196.

13. Lioret S, Touvier M, Lafay L, Volatier J-L, Maire B. Dietary and physical activity patterns in French children are related to overweight and socioeconomic status. J Nutr 2008;138:101-7.

14. Bowen L, Taylor AE, Sullivan R, et al. Associations between diet, physical activity and body fat distribution: a cross sectional study in an Indian population. BMC Public Health [Internet] 2015;15:281 Available at http://www.biomedcentral.com/1471-2458/15/281/abstract\%.

15. Food and Agricultural Organization. Human energy requirements: report of a Joint FAO/WHO/UNU Expert Consultation. FAO Food Nutr Tech Rep Ser [Internet] 2001;0:96 Available at http://www.fao.org/docrep/007/ y5686e/y5686e08.htm.

16. Fulton JE, Dai S, Steffen LM, Grunbaum JA, Shah SM, Labarthe DR. Physical activity, energy intake, sedentary behaviour and adiposity in youth. Am J Prev Med 2009;37:S40-9 Available at http://dx.doi.org/ 10.1016/j.amepre.2009.04.010.

17. Giammattei J, Blix G, Marshak HH, Wollitzer AO, Pettitt DJ. Television watching and soft drink consumption. Arch Pediatr Adolesc Med 2003;157: 882-6.

18. Suresh V, Rupnath K, Ramesh VR, MR, Sambasivarao KRS T. Television watching and sleep promotes obesity in urban and semi-urban children in India. J Toxicol Environ Health 2011;3:001-7.

19. Laxmaiah A, Nagalla B, Vijayaraghavan K, Nair M. Factors affecting prevalence of overweight among 12- to 17-year-old urban adolescents in Hyderabad, India. Obesity (Silver Spring) 2007;15:1384-90.

20. Khadilkar VV, Khadilkar AV, Cole TJ, Sayyad MG. Cross-sectional growth curves for height, weight and body mass index for affluent Indian children, 2007. Indian Pediatr 2009;46:477-89.

21. National Cancer Institute. Dietary assessment primer. Available at https:// dietassessmentprimer.cancer.gov/approach/table.html.

22. Kyle UG, Bosaeus I, De Lorenzo AD, et al. Bioelectrical impedance analysis-part II: utilization in clinical practice. Clin Nutr 2004;23: 1430-53.

23. Barbosa N, Sanchez CE, Vera JA, Perez W, Thalabard J-C, Rieu M. A physical activity questionnaire: reproducibility and validity. J Sports Sci Med [Internet] 2007;6:505-18 Available at http://www.ncbi.nlm.nih.gov/ pubmed/24149485\%5Cnhttp://www.pubmedcentral.nih.gov/articlerender. fcgi?artid $=$ PMC3794492.

24. Anthroplus WHO, Computers P. WHO AnthroPlus for Personal Computers Manual Software for Assessing Growth of the World $\hat{a} €^{\mathrm{TM}} \mathrm{s}$ Children. Available at www.who.int/childgrowth/software/en/.

25. Desai MP, Colaco P. Growth hormone deficiency and insensitivity In: Desai MP, Menon PSN, Bhatia Veds. Pediatric Endocrine Disorders 2nd edn Hyderabad, India: Universities Press (India) Pvt. Ltd, 2008: 188-92.

26. McCarthy HD, Cole TJ, Fry T, Jebb SA, Prentice AM. Paediatric highlight, body fat reference curves for children. Int J Obes 2006;30:598-602.

27. Rutishauser IHE. Dietary intake measurements. Public Health Nutr 2016;8: $1100-7$.

28. Guenther PM, Kott PS, Carriquiry AL. Community and international nutrition development of an approach for estimating usual nutrient intake distributions at the population level 1, 2. J Nutr 1997;127:1106-12.

29. C-Diet. C- Diet. Pune, India: Xenios Technology, 2012.

30. Chiplonkar SA, Agte VV. Extent of error in estimating nutrient intakes from food tables versus laboratory estimates of cooked foods. Asia Pac J Clin Nutr [Internet] 2007;16:227-39 Available at: http://www.ncbi.nlm. nih.gov/pubmed/17468077.

31. Kulkarni SA, Ekbote VH, Sonawane A, Jeyakumar A, Chiplonkar SA, Khadilkar AV. Beneficial effect of iron pot cooking on iron status. Indian J Pediatr [Internet] 2013;80:985-9 Available at http://link.springer.com/ 10.1007/s12098-013-1066-z.

32. Composition of Foods Raw, Processed, Prepared USDA National Nutrient Database for Standard Reference, Release 232010 September https://www. ars.usda.gov/ARSUserFiles/80400525/Data/SR23/sr23_doc.pdf. 


\section{Articles | Khadilkar et al.}

33. Gopalan C, Ramasastri BV, Balasubramanian SG. Nutritive Value of Indian FoodNational I. Hyderabad: (Indian Council of Medical Research; ICMR); A report of the expert group of the Indian Council of Medical Research. Nutrient requirement and recommended dietary allowances for Indians, 2009.

34. Centre for Disease Control and Prevention How much physical activity do children need? [Internet]. Available fat https://www.cdc.gov/physicalactivity/ basics/children/ (cited 25 October 2016).

35. World Health Organisation Available at http://www.who.int/childgrowth/ standards/weight_for_age/en/index.

36. Agarwal DK, Agarwal KN, Upadhyay SK, Mittal R, Prakash R, Rai S. Physical and sexual growth pattern of affluent Indian children from 5 to 18 years of age [review]. Indian Pediatr 1992;29:1203-82.
37. Cole TJ. A simple chart to identify non-familial short stature. Arch Dis Child 2000;82:173-6.

38. Atkin L, Davies PSW. Diet composition and body composition in preschool children 1, 2. Am Soc Clin Nutr 2000;72:15-21.

39. Janssen I, LeBlanc AG. Systematic review of the health benefits of physical activity and fitness in school-aged children and youth. Int J Behav Nutr Phys Act [Internet] 2010;7:40 Available at http://www.ncbi.nlm.nih.gov/ pubmed/20459784 (cited 2 January 2017).

40. Must A, Tybor DJ. Physical activity and sedentary behavior: a review of longitudinal studies of weight and adiposity in youth. Int J Obes (Lond) [Internet] 2005;29 ((Suppl 2)): S84-96 Available at http://www.ncbi. nlm.nih.gov/pubmed/16385758 (cited 2 January 2017]. 